\title{
An Agenda for Increasing Grant Funding of Emergency Medicine Education Research
}

\author{
Esther K. Choo, MD, MPH, Rosemarie Fernandez, MD, Emily M. Hayden, MD, MHPE, Jeffrey
}

I. Schneider, MD, Brian Clyne, MD, Shiphra Ginsburg, MD, MEd, and Larry D. Gruppen, PhD

\begin{abstract}
Funding is a perennial challenge for medical education researchers. Through a consensus process, the authors developed a multifaceted agenda for increasing funding of education research in emergency medicine (EM). Priority agenda items include developing resources to increase the competitiveness of medical education research faculty in grant applications, identifying means by which departments may bolster their faculty's grant writing success, taking long-term steps to increase the number of grants available to education researchers in the field, and encouraging a shift in cultural attitudes toward education research.
\end{abstract}

ACADEMIC EMERGENCY MEDICINE 2012; 19:1434-1441 (C) 2012 by the Society for Academic Emergency Medicine

$\mathrm{T}$ his article highlights the findings of the 2012 Academic Emergency Medicine (AEM) consensus conference breakout session on funding of education research in emergency medicine (EM). The objectives of the session were to 1) identify the major barriers to attainment of funding by EM education

From the Department of Emergency Medicine, Warren Alpert Medical School of Brown University (EKC, BC), Providence, RI; the Division of Emergency Medicine, University of Washington (RF), Seattle, WA; the Department of Emergency Medicine, Massachusetts General Hospital (EMH), Boston, MA; the Department of Emergency Medicine, Boston Medical Center, Boston University School of Medicine (JIS), Boston, MA; the Department of Medicine, Wilson Centre for Research in Education, Faculty of Medicine, University of Toronto (SG), Toronto, Ontario, Canada; and the Department of Medical Education, University of Michigan Medical School (LDG), Ann Arbor, MI. Breakout Participants: Michael Gisondi, Amy Kontrick, Nathan Kuppermann, Brandon Maughan, Douglas McGee, Craig Newgard, Mike Radeos, Mark Sahor, Kane Vacca, Adam Wilson, and Paul Zgyurzynski.

Received June 25, 2012; accepted June 5, 2012.

This manuscript is based on proceedings from the 2012 Academic Emergency Medicine consensus conference “Education Research in Emergency Medicine: Opportunities, Challenges, and Strategies for Success," held on May 9, 2012, in Chicago, IL.

The authors have no relevant financial information or potential conflicts of interest to disclose.

Supervising Editor: John Burton, MD.

Address for correspondence and reprints: Esther K. Choo, MD, MPH; e-mail: esther_choo@brown.edu. researchers, emphasizing factors demonstrated to correlate with funding success; 2) to discuss feasible strategies to overcoming identified barriers; and 3) to create an agenda for supporting and developing the funding success of medical education research faculty within EM. To encourage rapid adoption of our recommendations, the session participants opted to emphasize expansion of existing programs and workshops over creation of new programming whenever possible. Consensus participants recognized that any substantial change in grant funding is ultimately tied to changing the culture in which EM education research is viewed; therefore, action items for supporting a more global culture change were emphasized.

\section{THE CHALLENGE OF OBTAINING FUNDING FOR EM EDUCATION RESEARCH}

The Institute of Medicine has called for improved rigor of education research to support and integrate innovative medical education practices ${ }^{1}$; however, this directive lacks potency without a link to mechanisms for increasing available funding for education research. A paucity of funding has been identified as a major factor in the quantity and methodologic quality of medical education studies. ${ }^{2-4}$ The majority of education research-both within and outside of EM-is unfunded or underfunded. ${ }^{2,5,6}$ Existing funds are mostly from private foundations; education research has not been viewed as part of the traditional mission of the National Institutes of Health, the Canadian Institute for Health Research, or other federal institutions that support the majority of 
medical school research funding. While limited availability of education research grants is a significant hurdle to achieving funding, there are numerous barriers to obtaining funding for EM education researchers.

Dedicated administrative time directed to funding acquisition is a substantial barrier to funded education research activities. An EM educator who wishes to pursue research often has teaching and administrative roles in a residency program and/or medical school that leave little time for performing research and seeking funding. As funding is required to sustain meaningful and highquality research efforts, this dilemma becomes a vicious cycle. Education researchers may not be aware of opportunities for research funding or the steps required to successfully pursue grant support. Traditionally, education and research have been presented to academic clinicians as two mutually exclusive tracks. Research funding may be perceived to be something relevant only to basic scientists and clinical researchers. While those who plan for research-track careers usually begin contemplating funding opportunities early on, education researchers may not be as well informed about the process of pursuing grants. The very fact that much of education research is performed with little or no funding may perpetuate the concept that the process of seeking funding is not relevant to these researchers.

Educators who wish to pursue research may not receive the same mentorship and guidance to prepare them to be strong grant candidates, recognize appropriate grant opportunities, or prepare grant proposals. They may not be eligible for the same seed funds for acquiring pilot data or receiving opportunities for mentorship or collaboration, and they may not have departmental or institutional support for career development. These factors are all critical components to grant reviewers when determining the likelihood that an investigator will be able to successfully accomplish stated research goals.

The conundrum of medical education researchers is that receiving funding for education research may be hampered in part by the lack of existing rigorous EM education research. Published education research in EM is relatively rare. ${ }^{5-7}$ Issues common to published educational research include a lack of methodologic rigor, little improvement in educational outcomes, a narrow focus on learner satisfaction or other nonclinical goals, and small sample sizes and single-site designs. ${ }^{8,9}$ Aspiring researchers in EM may have little precedent to support proposals for education innovations with few examples of robust methodologic practices in designing education research projects. Perceptions of the lack of quality of EM education research projects may render funding entities reluctant to commit substantial resources to education researchers.

Changing the landscape for funding for education research may be challenging. To the extent that institutional support for pursuing funding influences the success of researchers, culture change may be a necessary component of increasing funded education research. Academic centers are known for prioritizing basic science and clinical research endeavors, as well as clinical care. Efforts to enhance education are sometimes seen as "subordinate" to other functions in the traditional academic medical center. ${ }^{10}$ Despite the presence of different advancement pathways within a university, faculty may feel that promotion processes prioritize research over teaching functions even when educators are performing research functions. Education researchers may be further hampered in success when competing for the few available funds by the status of EM as a relatively new field.

Factors associated with success in obtaining grant funding are well reported in the literature and include $\mathrm{in}^{11-16}$ 1) previous training in grant writing, 2) protected time to prepare a strong grant proposal, 3) existence of a peer review process, 4) a network of collaborators and mentorship support, 5) appropriate statistical and methodology support, and 6) the development of preliminary data and a track record of success. Additionally, the number of publications at the time of grant submission may affect overall success, whether directly or via the demonstration of adequate preliminary data and overall project feasibility. ${ }^{17,18}$

\section{AN AGENDA FOR INCREASING FUNDING FOR EM MEDICAL EDUCATION RESEARCH: CONSENSUS GOALS}

Increase Successful Grant Applications by EM

Education Researchers Through Training and

Mentorship

Small amounts of early career funding can markedly influence a junior faculty member's future research productivity. ${ }^{4,19}$ This influence is more pronounced when evaluating the effect of federally funded training grants. Unfortunately, the availability of such grants is limited, especially in the area of medical education research. ${ }^{11,19}$ Given the scarcity of such funding opportunities, it is likely that even a high-quality application may not result in funding. However, without a well-written, methodologically sound proposal, the chances of obtaining funding are virtually nonexistent. ${ }^{17,20}$

Training in Grant Writing. Formal training in the writing of grant applications is critical to success in obtaining funding. This observation has been well recognized in academic EM and has resulted in the creation of several programs designed to provide junior faculty with the skills necessary to initiate the creation of a grant proposal. The American College of Emergency Physicians (ACEP) offers the Emergency Medicine Basic Research Skills (EMBRS) workshop for junior faculty. This intensive course is designed to help individuals "become familiar with clinical research and outcomes, injury prevention, health care delivery, and effectiveness research" and includes introductory material on a number of research-related topics including grant writing. ${ }^{21}$ Additionally, the Society for Academic Emergency Medicine (SAEM) offers an annual workshop focused specifically on grant writing skills. ${ }^{22}$

While these workshops are largely focused on more traditional areas of basic and/or clinical research, as opposed to medical education research, the programs are not without value for those seeking education research guidance and expertise. There may be additional training or expertise needed to help focus skill development more toward medical education research 
methodology and proposal writing. ${ }^{23}$ The Association of American Medical Colleges Medical Education Research Certification (MERC) program offers a multifocused workshop on medical education research skills, although grant writing is not currently a focus of any one workshop. ${ }^{24-27}$ Solutions to the gap in medical education research-focused grant training could involve augmenting current programs and workshops to include specific tracks, expansion of medical education research programs (e.g. MERC) to include grant writing skills, or development of a novel grant writing training program.

Existing EM education fellowships should include training in grant writing for participants interested in education research careers with inclusion of a mentored grant writing experience. SAEM recently developed requirements for approved institutional EM research fellowships to include instruction in grant preparation, submission, and revision. ${ }^{28}$ To date, there are no education fellowships among the approved programs. ${ }^{29}$ Developing fellowship programs that meet the rigorous criteria with an emphasis on education research will help prepare medical education research faculty for grants acquisition, elevate the quality of research, and advance traditional perceptions of education researchers.

Mentorship. The need for development of a cohort of medical education research mentors is a consistent theme within the literature. Faculty should have grant applications routinely reviewed by one or more colleagues with content expertise and grant writing knowledge to ensure an accurate presubmission critique. ${ }^{12,15}$ In medical education research, finding easily accessible experts within the applicant's home institution can be challenging. ${ }^{23}$ Despite the ease of Internet-based communication, locating a medical education research expert to review a grant application can be a daunting task. These challenges increase when junior faculty compete for federal mentored career development grants ( $\mathrm{K}$ awards), where the active involvement of a federally funded mentor is critical to funding success. ${ }^{13}$

We propose two mechanisms to support mentorship through the grant writing experience. First, we recommend that the academic EM community seek experts in medical education research to form a committee or consultation service aimed toward providing presubmission grant reviews. Second, we suggest the development of a senior medical education research faculty database, enabling junior faculty to be matched with those more senior or experienced in medical education research. This development would likely require targeting individuals outside of EM. However, as noted by Gruppen, ${ }^{23}$ collaboration between clinician educators and medical education research experts is a mutually beneficial relationship that is increasingly critical as funding sources become more limited. Both initiatives would not only support early medical education research projects, but would also serve to establish a record of collaboration for the junior investigator. ${ }^{30}$

Increase Departmental and Institutional Support for Medical Education Research Faculty

Significant departmental and institutional buy-in is needed for success in grant funding, especially for
K-series grants or other similar training awards. Faculty workshops, development of interinstitutional collaborations, and preproposal reviews all require some element of support. Medical education research faculty may wish to take courses or pursue advanced degrees to attain research skills and increase their chances of success for research awards. Additionally, protected time for grant writing and development of preliminary data is often cited as a major factor relating to successful grant submissions. $^{12,14,30,31}$

Offering protected time for faculty in a department with high clinical demands can be a challenge, particularly as medical education research faculty seek K-type awards or other similar training grants. However, faculty may collaborate with departmental leaders to link their support to clear expectations for productivity and funding within a discrete time period.

Faculty pursuing education research will require access to expertise in qualitative and quantitative research design, measurement, and data analysis specifically applicable to medical education. Individual EM departments may not have the resources to support this level of specialization and knowledge, however. Institutional commitment to provide access to highly trained medical education research experts in the form of a center or independent department of medical education research can markedly enhance the productivity and success of faculty in clinical departments. ${ }^{32}$ Such centralized resources also strengthen the evidence of institutional support necessary to obtain competitive federal grants.

Department leaders may provide nonmonetary support for education researchers by serving as vocal advocates for the role of educators on grant teams. EM educators may be promoted as potential coinvestigators in research grants containing educational initiatives or critical process measures related to educational goals. Department chairs and research directors may advance their education researchers by highlighting achievements of educators and by promoting their education researchers as "translation experts." Such roles might increase the sophistication of the grant proposal, the feasibility of implementation of new practices by clinical staff, and the impact of the research innovation.

\section{Increase Interdisciplinary, Interinstitutional, and Interprofessional Education Research Collaboration} Collaboration is critically important for grant proposal development, as well as for the enhancement of the rigor of proposed studies within a grant application. Multi-institutional, multidisciplinary, and multiprofessional studies often involve larger sample sizes, more generalizable results, and research teams with a broader base of knowledge and expertise. ${ }^{33-35}$

Topics that are common to many specialties (e.g., trainee competency assessment, procedural training) are potential targets of multidisciplinary investigations and may allow modest funds from a variety of sources to be leveraged into higher impact studies. For example, EM faculty may broaden their focus from "emergency care" to "acute care" topics, thereby leading to collaborations with departments of surgery or medical intensive care. Finally, collaborative physician-nursing grants are also 
increasingly receiving funding as interprofessional efforts.

Developing and maintaining such research collaborations requires significant initial investment of time and effort to establish a data repository, a project planning platform, and a resource sharing plan. In a review of a small grants program, El-Sawi et al. ${ }^{4}$ demonstrated that the presence of funding directly affected the number of collaborations within a project, as well as the likelihood of continuing collaborative relationships beyond the period of the project. A simple edict to "improve collaboration" without associated support is unlikely to truly improve medical education research quality and quantity.

One approach to overcoming the expense and energy required for an individual to build national collaborations may be for the specialty to develop a common national network for EM education research. Education researchers might also utilize existing national and international EM research networks (e.g., PECARN, EMNet).

\section{Increase the Awareness and Accessibility of Existing} Funding Mechanisms for Education Research

Given the challenges of obtaining funding for medical education research, it is imperative that investigators become aware of the existing funding sources, especially those with a record of supporting education projects in EM. Finding an appropriate grant mechanism for a specific project can be a significant obstacle to initiating the process of seeking funds. This need has been recognized by several commercial services that routinely scan funding agencies and foundations for requests for proposals and statements of funding goals. Much of this work is then organized into searchable databases. Examples of these entities include the Community of Science (http://www.cos.com/), a comprehensive resource with over 400,000 funding opportunities, worth over $\$ 33$ billion, and the Foundation Center (http://www.foundationcenter.org/). Many of these services require a subscription fee, which may be prohibitive for some institutions.

There are free searchable database services, such as grants.gov (http://www.grants.gov/), where all federal funding opportunities, including those available through the National Institutes of Health and National Science Foundation, can be found. Others include the Agency for Healthcare Research and Quality Grants On-Line Database (www.gold.ahrq.gov), which is searchable by project type, focus area, keyword, and geographic location. A number of specialty societies and organizations maintain lists of grant opportunities relevant to the specialty or group. These lists are not always up to date or comprehensive, but may serve as affordable alternatives to commercial services.

Several national EM specialty organizations (ACEP, SAEM, the Council of Emergency Medicine Residency Directors [CORD], the Emergency Medicine Residents Association, and the Emergency Medicine Foundation [EMF]) offer grants with a history of funding medical education research. Many of these program details and application processes are not available from a central online resource. Creating a specialty-specific, centralized database of funding sources could assist EM education researchers to identify potential grant opportunities that match their areas of interest. This funding resource could include the various private foundations and federal agencies that provide the majority of medical education research funding.

A centralized resource, such as a dynamic website that allowed those with successful funding from lesserknown foundations to edit information, would render a more accurate and relevant resource. Such a medical education research funding resource might be created, promoted, and maintained as part of the duties of a task force or research collaborative.

Ideally, any resource pointing to specific sources of funding would also include information and advice about navigating the application process, and linkage to faculty successful in obtaining medical education research funding by a given organization, to create opportunities for direct mentorship that will increase the accessibility of the process to junior faculty. Demystifying the process and creating opportunities for direct mentorship is especially important for junior faculty whose success with small grant programs will increase the likelihood that a grant review committee will accept a future grant.

\section{Increase Sources of Funding for Education Research} While the majority of this breakout session was dedicated to increasing the competitiveness and skills of EM faculty in pursuing existing grants, another avenue to funding would be to increase the supply of funding mechanisms for education research. Long-term, national collaborative EM education research networks may seek to independently raise funds to establish grant programs. Education researchers in EM and other fields may lobby national (federal or private) organizations to increase programs dedicated to education innovations. Education leaders may also seek large-scale institutionbased innovations modeled after the example of many academic centers across the country such as Dartmouth, Johns Hopkins, the University of California-San Francisco, and the University of Michigan. ${ }^{32,36-38}$ Such programs, which provide direct and indirect support of medical education research faculty, including funds for protected research time, seed funds, travel to scholarly conferences, resources for data collection and analysis, and even secretarial support, essentially serve as minigrants that allow faculty to be productive and more likely to succeed in future efforts to obtain extramural grant funding.

More immediately, EM education researchers may seek to increase the attention given to education research within funding programs supported by our own specialty. EM grant programs are few, but include the ACEP/EMF grants, SAEM grants, and CORD grants. With the exception of a single CORD grant, these programs do not currently provide funding directed to education research. EM education leaders are well positioned to advocate for education researchers to be invited to apply or to serve as coinvestigators on existing grant opportunities and to advocate for creation of additional grants specifically for medical education research projects. Importantly, medical education research applications for current EM-specific funding sources must 1) be reviewed by appropriate 


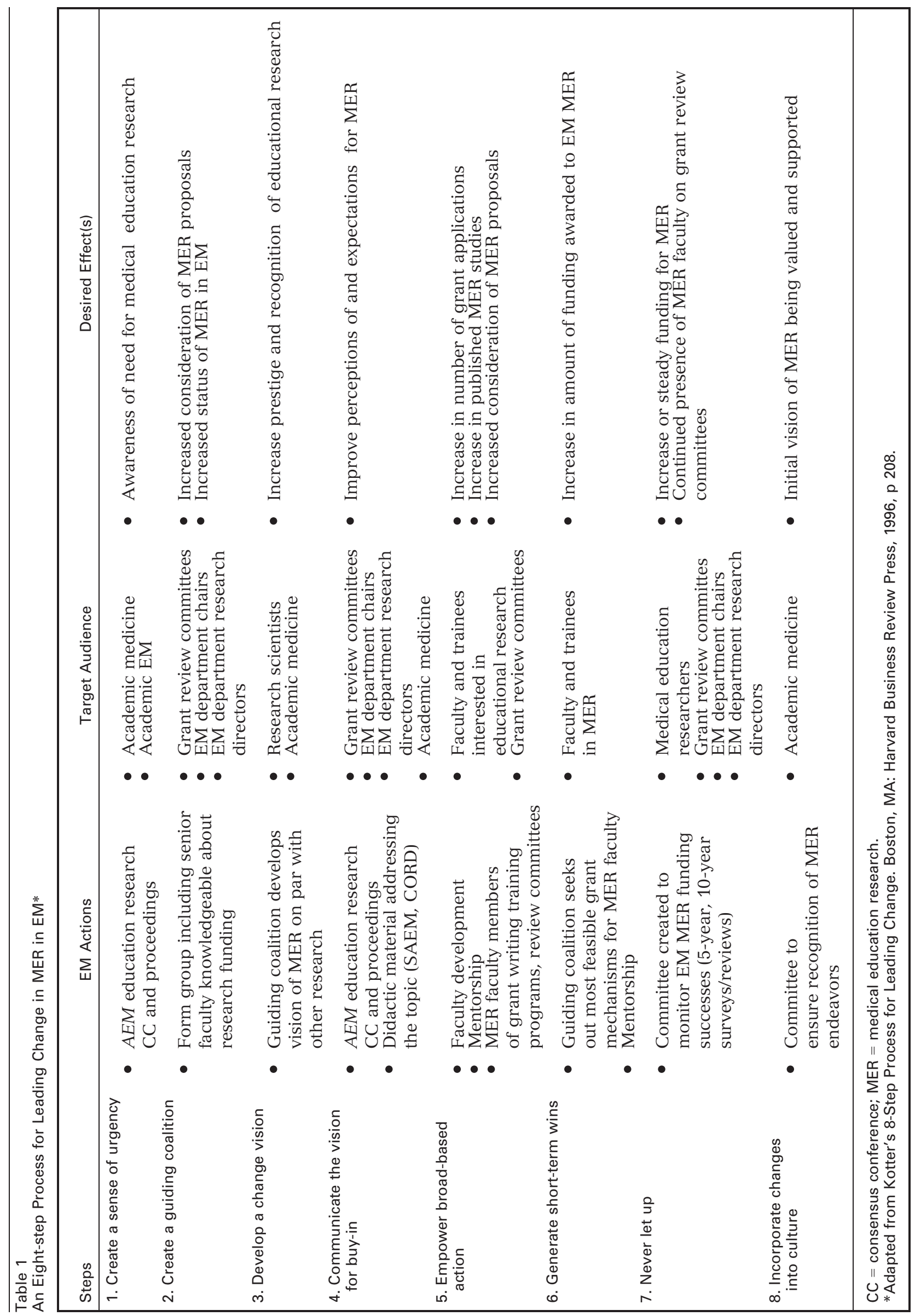




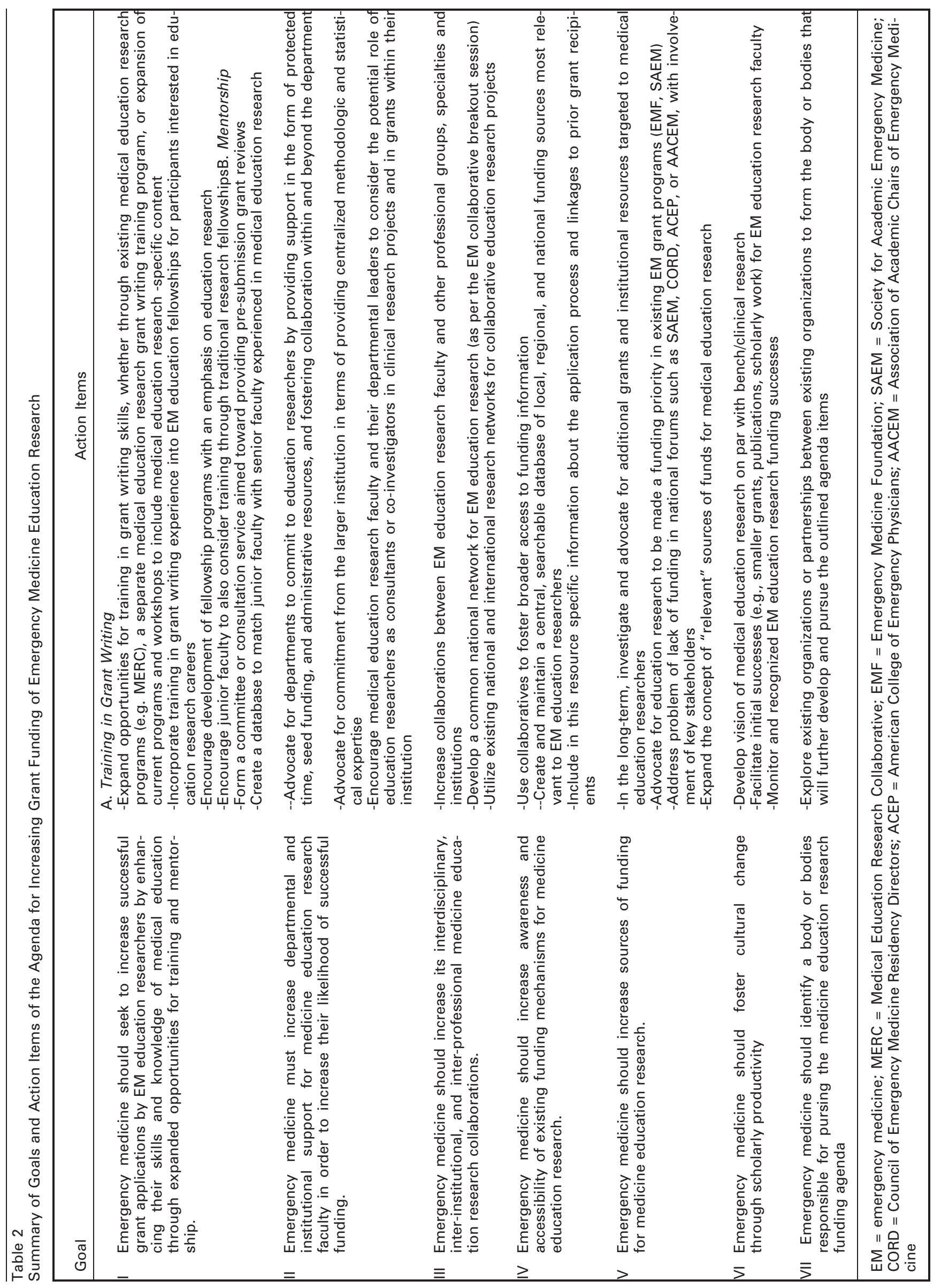


medical education research experts for methodologic rigor and, 2) if methodologically sound, must be considered as having comparable value to clinical and basic science submissions. An increase in the number and quality of applications related to EM education research may signal the importance of increasing the budget committed to medical education research projects in the future.

Supporting education research should become an explicitly stated goal of our national EM organizations. The consensus group recommends the creation of a task force for investigating and advocating for avenues to increase funding for education research or inclusion of education researchers in established grant funds.

Most immediately, education researchers may begin to expand to other relevant sources of funds for medical education research. Recent political, regulatory, and educational emphasis on patient safety, cost-effective care, and pay-for-performance metrics suggests that these avenues may be productive approaches to educational research funding. Education researchers should be encouraged to seek funding within broader areas that overlap with education research and not limit their grant applicants to those explicitly identified as "educational research grants." Institutional training grants (such as the newly funded National Heart, Lung and Blood Institute Career Development Programs in EM) may provide another, relatively accessible avenue for funding and should be considered by junior education research investigators who may be able to apply their skills to the specific content areas supported by these grants.

\section{Foster Cultural Change}

While increasing the quality of grant applications and study designs will improve the success of funding educational research, the breakout session participants emphasized that a worthwhile overarching goal would be to create culture change so that medical education research is considered of equal merit to basic science and clinical research. The low prestige of education research in academic medicine has unfortunate effects, ranging from dissuading nascent researchers from pursuing education research to biases of grant review committees against education research projects. A future vision of medical education research is one in which education researchers have the tools necessary to competitively apply for grants, and grant review committees give the same consideration to educational research proposals. To change the current culture and perception of medical education research as a "soft" field of research, the authors propose a multifaceted approach, using Kotter's Eight-step Process for Leading Change ${ }^{39}$ (Table 1) as a framework for potential activities.

\section{Identify a Body or Bodies Within EM to Pursue Medical Education Research Funding-related Action Items}

The breakout session discussed the pursuit of an agenda for increasing successful funding of EM education research. Although this discussion did not result in the identification of a specific body or structure (e.g., task force vs. committee vs. collaborative) to pursue the outlined medical education research funding agenda, participants agreed that such a body should be created or that the agenda should be formally pursued by interested members of existing organizations such as SAEM, CORD, and the Clerkship Directors in EM.

\section{CONCLUSIONS}

Through a consensus process, we developed a multifaceted agenda for increasing funding of education research in EM (see full list of goals with individual actions items in Table 2). The agenda includes developing resources to increase the competitiveness of medical education research faculty in grant applications, identifying means by which departments may bolster their faculty's grant writing success, taking long-term steps to increase the number of grants available to medical education research researchers in our field, and encouraging a shift in cultural attitudes toward education research.

\section{References}

1. Kohn LT (ed). Academic Health Centers: Leading Change in the 21st Century. Washington DC: National Academies Press, 2004.

2. Reed DA, Kern DE, Levine RB, Wright SM. Costs and funding for published medical education research. JAMA. 2005; 294:1052-7.

3. Reed DA, Cook DA, Beckman TJ, et al. Association between funding and quality of published medical education research. JAMA. 2007; 298:1002-9.

4. El-Sawi NI, Sharp GF, Gruppen LD. A small grants program improves medical education research productivity. Acad Med. 2009; 10(Suppl):S105-8.

5. Kuhn GJ, Shayne P, Coates WC, et al. Critical appraisal of emergency medicine educational research: the best publications of 2009. Acad Emerg Med. 2010; 17(Suppl 2):S16-25.

6. Shayne P, Coates WC, Farrell SE, et al. Critical appraisal of emergency medicine educational research: the best publications of 2010. Acad Emerg Med. 2011; 18:1081-9.

7. Farrell SE, Coates WC, Khun GJ, et al. Highlights in emergency medicine medical education research: 2008. Acad Emerg Med. 2009; 16:1318-24.

8. Dauphinee WD, Wood-Dauphinee S. The need for evidence in medical education: the development of best evidence medical education as an opportunity to inform, guide, and sustain medical education research. Acad Med. 2004; 79:925-30.

9. Prideaux D. The emperor's new clothes: from objectives to outcomes. Med Educ. 2000; 34:168-9.

10. Shahjahan RA. An exploration of the effects of funding cutbacks to Ontario medical schools: privileging research over education. Higher Educ Perspec. 2005; 1:56-75.

11. Carline JD. Funding medical education research: opportunities and issues. Acad Med. 2004; 79: 918-24.

12. Koppelman GH, Holloway JW. Successful grant writing. Paediatr Respir Rev. 2012; 13:63-6. 
13. Brock MV, Bouvet M. Writing a successful NIH Mentored Career Development Grant (K award): hints for the junior faculty surgeon. Ann Surg. 2010; 251:1013-7.

14. Cole SS. Researcher behavior that leads to success in obtaining grant funding: a model for success. Res Manage Rev. 2006; 15:1-16.

15. Inouye SK, Fiellin DA. An evidence-based guide to writing grant proposals for clinical research. Ann Intern Med. 2005; 142:274-82.

16. Ebong I. Relating capacity to a faculty funding persistence profile. SRA J. 1999; 31:23-34.

17. Carden DL, Dronen SC, Gehrig G, Zalenski RJ. Funding strategies for emergency medicine research. Acad Emerg Med. 1998; 5:168-76.

18. Simons-Morton DG. Funding avenues for research in emergency medicine at the National Institutes of Health and the National Heart, Lung, and Blood Institute. Acad Emerg Med. 1996; 3:202-4.

19. Albanese M, Horowitz S, Moss R, Farrell P. An institutionally funded program for educational research and development grants: it makes dollars and sense. Acad Med. 1998; 73:756-61.

20. Reif-Lehrer L. Writing a Successful Grant Application. Boston, MA: Jones and Barlett, 1989.

21. American College of Emergency Physicians. Emergency Medicine Basic Research Skills (EMBRS) Workshop. Available at: http://www.acep.org/embrs/. Accessed Sep 9, 2012.

22. Society for Academic Emergency Medicine. SAEM Membership Benefits. Annual Meeting: Intensive Grant Writing Workshop. Available at: http://www. saem.org/saem-membership-benefits. Accessed Sep 9, 2012.

23. Gruppen LD. Improving medical education research. Teach Learn Med. 2007; 19:331-5.

24. Association of American Medical Colleges. Medical Education Research Certificate (MERC) Program. Available at: https://www.aamc.org/members/gea/ merc/. Accessed Sep 9, 2012.

25. Coates WC, Love JN, Santen SA, et al. Faculty development in medical education research: a cooperative model. Acad Med. 2010; 85:829-36.

26. Love JN, Coates WC, Santen SA, et al. The MERC at CORD Scholars Program in medical education research: a novel faculty development opportunity for emergency physicians. Acad Emerg Med. 2009; 16(Suppl 2):S37-41.

27. Gruppen LD, Yoder E, Frye A, Perkowski LC, Mavis B. Supporting medical education research quality: the Association of American Medical Colleges' Medical Education Research Certificate program. Acad Med. 2011; 86:122-6.

28. Society for Academic Emergency Medicine. SAEM Institutional Research Fellowship Program. Available at: http://www.saem.org/institutional-researchfellowship-program. Accessed Sep 9, 2012.

29. Society for Academic Emergency Medicine. SAEM Research Fellowship Directory. Available at: http:// www.saem.org/fellowship-directory. Accessed Sep 9, 2012.

30. Biros MH, Barsan WG, Lewis RJ, Sanders AB. Supporting emergency medicine research: developing the infrastructure. Acad Emerg Med. 1998; 5: 177-84.

31. Rangel SJ, Moss RL. Recent trends in the funding and utilization of NIH career development awards by surgical faculty. Surgery. 2004; 136:232-9.

32. Gruppen LD. The Department of Medical Education at the University of Michigan Medical School: a case study in medical education research productivity. Acad Med. 2004; 79:997-1002.

33. Cook DA, Andriole DA, Durning SJ, Roberts NK, Triola MM. Longitudinal research databases in medical education: facilitating the study of educational outcomes over time and across institutions. Acad Med. 2010; 85:1340-6.

34. O'Sullivan PS, Stoddard HA, Kalishman S. Collaborative research in medical education: a discussion of theory and practice. Med Educ. 2010; 44: 1175-84.

35. Shea JA, Arnold L, Mann KV. A RIME perspective on the quality and relevance of current and future medical education research. Acad Med. 2004; 79: 931-8.

36. Nierenberg DW, Carney PA. Nurturing educational research at Dartmouth Medical School: the synergy among innovative ideas, support faculty, and administrative structures. Acad Med. 2004; 79: 969-74.

37. Thomas PA, Wright SM, Kern DE. Educational research at Johns Hopkins University School of Medicine: a grassroots development. Acad Med. 2004; 79:975-80.

38. Irby DM, Hodgson CS, Muller JH. Promoting research in medical education at the University of California, San Francisco, School of Medicine. Acad Med. 2004; 79:981-4.

39. Kotter JP. Leading Change. Boston, MA: Harvard Business Review Press, 1996, p 208. 